\title{
Monitoring a Micromechanical Process in Macroscale Carbon Nanotube Films and Fibers
}

\author{
By Wenjun Ma, Luqi Liu, Rong Yang, Taihua Zhang, Zhong Zhang, Li Song, \\ Yan Ren, Jun Shen, Zhiqiang Niu, Weiya Zhou, and Sishen Xie*
}

Because of their promising mechanical properties and low density, carbon nanotubes (CNTs) have been widely envisioned as ideal building blocks for high performance engineering materials since the their discovery. Theoretical and experimental work on the mechanical properties of individual or bundles of CNTs proceeded well at the end of the last century. ${ }^{[1-3]}$ It is now widely held that the modulus and strength of a typical single-walled carbon nanotube (SWNT) can reach 640 and $37 \mathrm{GPa}$, respectively, which surpass those of all existing materials. ${ }^{[4]}$ However, because of weak intertube interactions, it is still a great challenge to acquire such super mechanical properties when CNTs are assembled as macroarchitectures (such as fibers and films). For example, prior to 2007, the reported tensile strengths of continuous CNT fibers without a coagulant lay in the range from 0.4 to $1.2 \mathrm{GPa}$, no matter if they were spun from a suspension. ${ }^{[5-12]}$

Recently, Windle et al. reported the synthesis of continuous high-performance CNT fibers with the highest strength value of $9 \mathrm{GPa}^{[12]}$ This encouraging breakthrough enlightens the use of macroscale CNTs fibers as a structural reinforcement for many applications. They pointed out that the obtained tensile strengths depended on the gauge lengths of the tested specimens: when the gauge length increased from 1 to $10 \mathrm{~mm}$ (a typical value for the tensile tests of CNT fibers), the average strength rapidly dropped to $1 \mathrm{GPa}$. Such degradation was attributed to a load transfer

[*] Prof. S. S. Xie, W. Ma, Prof. W. Zhou, Y. Ren, J. Shen, Z. Niu Beijing National Laboratory of Condensed Matter Institute of Physics, Chinese Academy of Sciences Beijing 100190 (P. R. China)

E-mail: ssxie@aphy.iphy.ac.cn

W. Ma, Y. Ren, J. Shen, Z. Niu

Graduate School of the Chinese Academy of Sciences Beijing 100080 (P. R. China)

Dr. L. Liu, Prof. Z. Zhang

National Center for Nanoscience and Nanotechnology Chinese Academy of Sciences

Beijing 100190 (P. R. China)

Prof. T. H. Zhang, R. Yang

State Key Laboratory of Nonlinear Mechanics (LNM)

Institute of Mechanics, Chinese Academy of Sciences Beijing 100190 (P. R. China)

Dr. L. Song

Center for Nanoscience and Department für Physik

Ludwig-Maximilians-Universität München

Geschwister-school-platz 1

80539 München, Germany

DOI: 10.1002/adma.200801335 deficiency caused by local defects during densification. In fact, poor load transfer does not only come from the weak intertube connections, but also from the enlargement of the failure possibility when load is transferred through a long gauge length or large cross section area. Up to now, the attention paid to this type of 'negative' size effect seems inadequate. This inadequateness is reflected in the usually missed mention of the sizes of the specimens when the experimental results are referred to, and makes systematically comparative studies difficult. Besides the size effect, the variety of CNT constructions in specific macrostructures and the difficulty of accurately measuring their volume fraction in fibers also blur the evaluation of the CNT fibers' mechanical properties. Thus, we need a generic methodology independent of mechanical measurements to help us explore the inherent interbundle strength and microscopic failure process for various CNT macroarchitectures.

As a nondestructive and readily available measurement, Raman scattering has been widely used to identify CNTs' diameters or alignment degree. ${ }^{[13,14]}$ Moreover, since Raman scattering is sensitive to the inter-atomic distance, when CNTs are mechanically strained, there is a linear relationship between the Raman peaks' shift and local strain. ${ }^{[15,16]}$ Wagner's group has demonstrated a successful case of utilizing CNTs as stress sensors in composite materials. ${ }^{[17]}$ In this report, we introduce Raman measurements to strained macroscale SWNT films and fibers. By investigating the variation of the $G$ ' band under strain, we can infer the structural deformation process of the micrometer-scale SWNT networks, and further give prediction of the macrostructures' moduli. Together with systematic tensile tests, we could elucidate the influence of CNT fibers' geometries on their mechanical performance.

The SWNT films used are directly synthesized by chemical vapour deposition (CVD), and detailed information can be found in our earlier work. ${ }^{[18]}$ Since the fabricated films are mechanically anisotropic, we can easily slice a strip from them and twist it into a few-centimeters-long fiber. Figure 1 shows a typical twisting process. Compared with the existing spinning methods developed by other groups, ${ }^{[5-11]}$ our twisting method may not be suitable for continuous production of CNT fibers, but the control of the diameters and twisting degrees is more convenient. So the fabricated fibers are ideal systems for the study of CNT macroarchitectures.

Typical $G^{\prime}$ band Raman spectra for unstrained and maximum-strained films and fibers are shown in Figure 2a. The downshifting trend of the peak position and the asymmetrical broadening of the line shape after strain are the major characteristics of the Raman spectra for both types of specimens. 


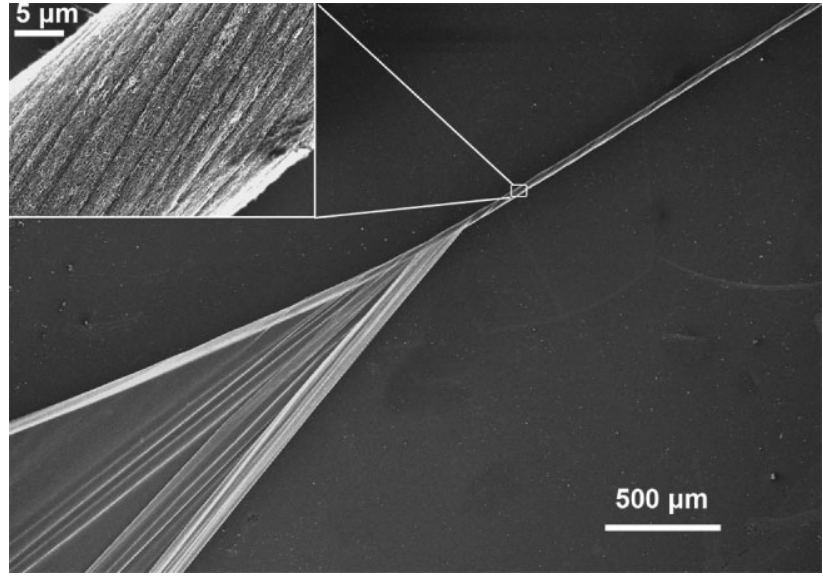

Figure 1. The SEM image shows a piece of as-grown film with a width of $2 \mathrm{~mm}$ and a thickness of $200 \mathrm{~nm}$ being twisted into a fiber. It should be pointed out that, for the specimens prepared for tensile tests, there still is a densification procedure before twisting by immersing the films in acetone. This procedure effectively reduces the free volume in the fibers and is necessary for the later comparison of mechanical properties between fabricated fibers and films (whose thicknesses are measured after densification). a

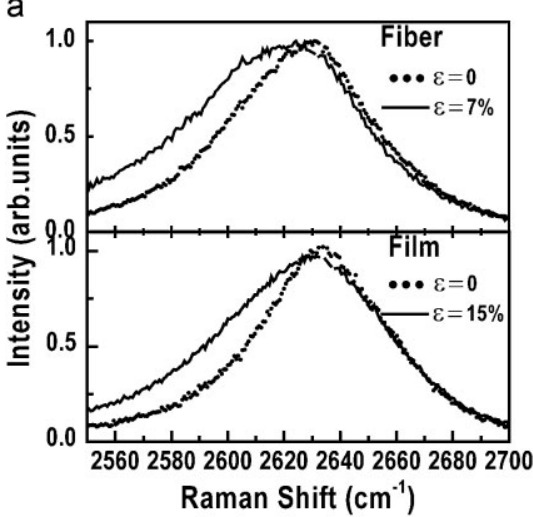

C

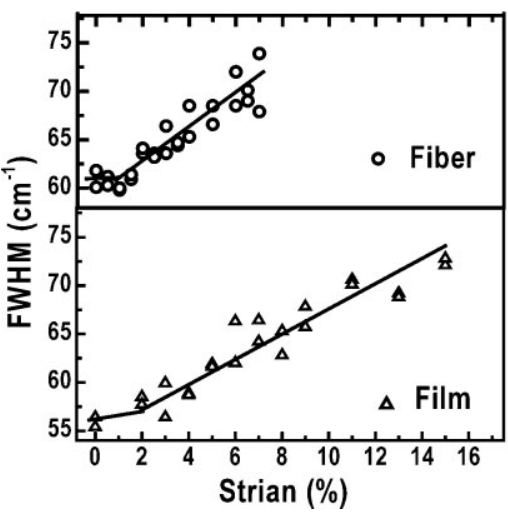

b

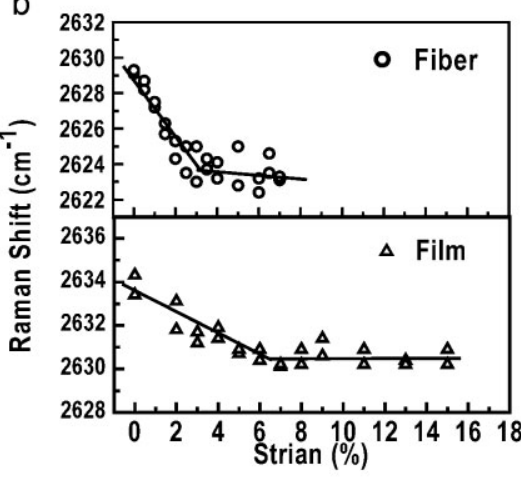

d

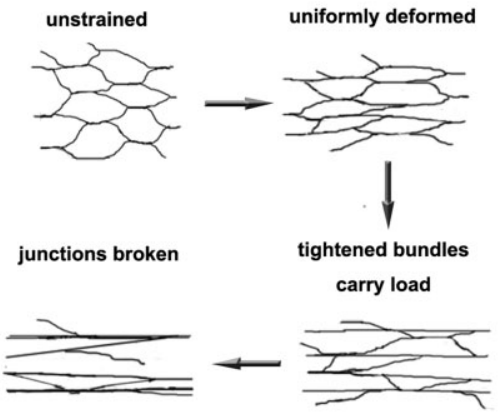

Figure 2. Typical $G^{\prime}$-band Raman spectra of a strained SWNT film and fiber. a) Comparison of the Raman spectra for unstrained and max-strained film and fiber. b) The Raman shift of the $G^{\prime}$ band as a function of applied strain. c) The widths of the $G^{\prime}$ band for a strained film and fiber. d) A schematic image shows the deformation process for the SWNT meshes in a film when macroscale strain is applied. The deformation process in our SWNT fiber is similar except for the pre-elongation of the meshes as a result of the twisting process.
The downshifts of the $G^{\prime}$ band are expected, and arise from the weakening of the carbon-carbon bonds as a result of the elongated inter-atomic distance. Asymmetrical $G^{\prime}$-band line shapes under strain are uncommon and have not been reported in the case of individual strained CNTs. Moreover, when more Raman measurements are performed at different strains (Fig. 2b), the downshifts of the $G^{\prime}$ band show two-stage features: at low strains, they increase linearly with a rate of 0.65 and $1.7 \mathrm{~cm}^{-1}$ per $1 \%$ strain for the film and fiber, respectively; once the strains exceed a certain point ( $6 \%$ for the film and $2.5 \%$ for the fiber), the peak position of the $G^{\prime}$ band plateaus until the final breakage of the specimens. As to the broadenings (shown in Figure $2 \mathrm{c}$ ), they start at $2 \%$ and $1 \%$ for the film and fiber, respectively, and linearly increase with strain until the final breakage of the specimens.

Such variation of the Raman spectra reflects the microscopic deformation process in strained CNT macroarchitectures. In our earlier work, we proposed that the SWNT bundles self-assemble into mesh-like structures as the basic units of the macroscale film. ${ }^{[18]}$ Figure $2 \mathrm{~d}$ illustrates the deformation process of the SWNT meshes in the strained film: when strain is gradually applied to the films, the meshes are first deformed to longer and narrower structures until some bundles are completely tightened; the overloaded junctions then begin to fail and local stress is redistributed to neighboring meshes; finally only the strongest junctions carry the loads before the breakage of the films or fibers. By analyzing the results of the Raman tests, we can monitor the micromechanical process in macroscale CNT films and fibers by three aspects as below.

First, the value of the downshift rate of the $G^{\prime}$ band with respect to strain at the low-strain stage gives us a quantitative sense of how much macroscale strain comes from the axial extension of CNTs. As shown in Figure 2d, at the low-strain stage, macroscale strain of the specimen mainly arises from the uniform deformation of the meshes rather than axial extension of the bundles. According to Corning's report, the average downshift rate of the $G^{\prime}$ band for strained individual SWNTs is $37.5 \mathrm{~cm}^{-1}$ per $1 \%$ strain, ${ }^{[16]}$ which is 56 times and 21 times higher than that of our films and fibers, respectively. Such small downshift rates in our case imply that SWNTs' axial extensions merely contribute several percent to the total macroscale strain, which results in the remarkable drop of the macroarchitecture moduli compared with that of the individual CNTs.

Second, the final downshifts of the $G^{\prime}$ band peak indicate the general strength level of the interbundle junctions. For example, in the case of our film, the downshift of the $G^{\prime}$ band reaches to $4 \mathrm{~cm}^{-1}$ at a strain of $6 \%$ and then plateaus even though more strain is applied. This transition implies that the loads applied to the meshes have reached the average bear limit 
of the junctions. Further added load will no longer be homogenously carried by all CNT bundles but by only a part of straight bundles that belong to strong meshes.

Third, the asymmetric broadening of the $G^{\prime}$ line shape provides much information about the stress distribution in the CNT macroarchitecture. Take the SWNT film in Figure 2a and 2c as example, the broadening of the $G^{\prime}$ band starts at a strain of $2 \%$, and almost all broadening comes from the low-wavenumber edge. This illustrates that before the universal breakage of meshes, some bundles have begun to carry more load than others. Following the increased strain applied on the film, the width increases linearly until the final breakage of the specimen. This trend together with the plateau stage of the downshifting clearly demonstrates the load at large strain is mainly carried by part of the bundles in the film. Such a strength distribution accelerates the stress concentration and lowers the strength of the CNT macroarchitectures.

In Figure 2, the variation trends of the Raman $G^{\prime}$ band spectra for the strained films and fibers are obviously different. The differences result from the twisting structure of the fibers. In 2004, Baughman's group improved the Fan's array-spinning technique, and realized the importance of the twisting process for the synthesis of macroscale CNT fibers. ${ }^{[4]}$ They stated that the twisted CNTs generated lateral force, which then gave rise to nanometer-scale friction force to couple these tens-of micrometers-long CNT bundles together as a continuous fiber. After that, the enhancement effects of the twisting structures to the fibers' mechanical properties were also verified by some other researchers. ${ }^{[19,20]}$ However, because of the shortage of easily available samples and the limitation of macroscale tensile tests, we know little about how the friction law works at the nanometer scale, and how the parameters such as twisting angle and twisting force influence the mechanical performance of these spun CNT fibers. Here, with the Raman data and the tensile test results, we could illustrate how the twisting-induced structure deformation improves the load transfer and strengthens the interbundle connections.

First, the twisting process results in the pre-elongation of the meshes, which is reflected by the downshift of the $G^{\prime}$ band for unstrained fibers compared with unstrained films. Figure 3

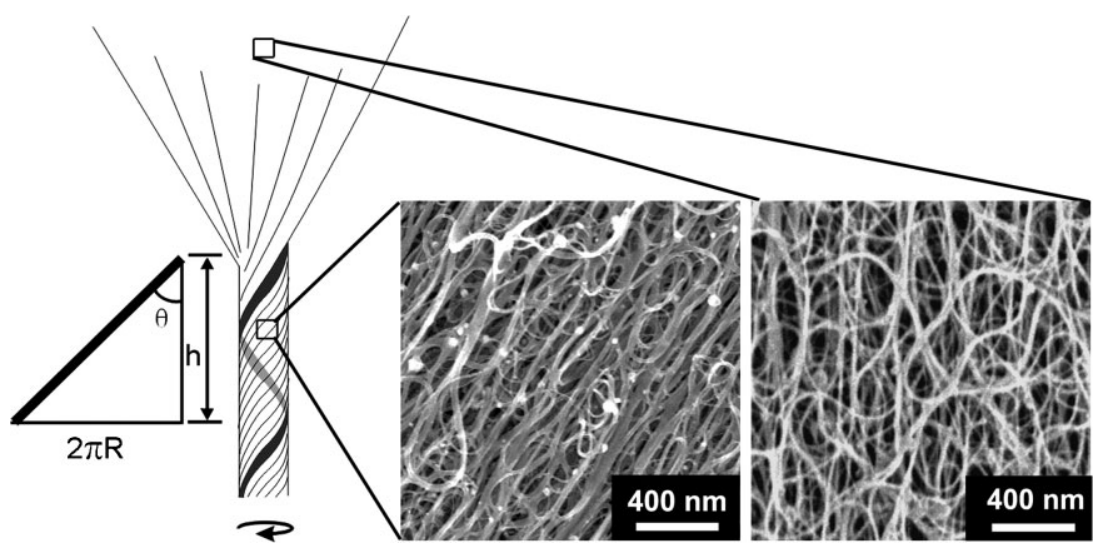

Figure 3. The schematic image left illustrates the twisting process and shows the geometrical elongation for the film near the surface of the fiber. The two SEM images right come from the unstrained and strained part of the film, respectively. illustrates the twisting process. When a film is twisted into a fiber, the part near the center of the fiber undergoes a few twists and collapses into a cylindrical core, while the part on the surface of the fiber is forced to extend by a factor of $1 / \cos \theta$ to match the length of the center part. At a certain twist angle of $20^{\circ}$ (typical value for fibers with a diameter of $35-40 \mu \mathrm{m}$ ), the pre-extension of the film on the fiber's surface is $6.5 \%$, which will result in the Raman downshift of $4 \mathrm{~cm}^{-1}$ according to the film's downshift rate. In Figure $2 b$, the peak of the $G^{\prime}$ band of the unstrained SWNT fiber locates at $2629 \mathrm{~cm}^{-1}$, which downshifts by about $4 \mathrm{~cm}^{-1}$ compared with that of the unstrained film. Such accordance shows that the twisting-induced pre-elongation of the meshes are well embodied by the Raman results.

Because of the pre-elongation of the meshes, CNT bundles in the fibers are pre-tightened and helically aligned along the fibers' axis. So the strain of the fibers could be more effectively transferred to the axial elongation of the bundles, and the moduli of the fibers are enhanced as a result of the twisting process. Tensile tests show that the moduli of our CNT fibers range from 9-15 GPa, which is more than two times that of the films. Such enhanced load transfer efficiencies are clearly demonstrated by the $G^{\prime}$ band's downshift rate: the downshift rate at low strain for the fiber is 2.6 times of that for the film. In fact, we can further determine a numerical relationship between the obtained downshift rates and the moduli of the macroscale films or fibers. We define the strain transfer factor (STF) as the ratio of the obtained downshift rate for a strained fiber or film to the average downshift rate of strained individual CNTs. The modulus of the CNT macroarchitecture can then be deduced by a simple formula (detailed derivation can be found in the supporting information):

$E_{\text {macro }}=c \alpha\left\langle\cos ^{2} \beta\right\rangle E_{\text {tube }}$

Where $\alpha$ is the STF, $c$ is the volume fraction of the CNTs in the films or fibers. $E_{\text {tube }}$ is the average modulus of individual CNTs, here we adopt $640 \mathrm{GPa}^{[4]} \beta$ is the orientation angle for an arbitrary SWNT bundle in a film or fiber with respect to the tensile axis, and $\left\langle\cos ^{2} \beta\right\rangle$ describes the contribution of all inclined tubes to the modulus of the macroscopic sample. In 2D or $3 \mathrm{D}$ systems, complete random orientation gives $\left\langle\cos ^{2} \beta\right\rangle$ a value of $1 / 2$ or $1 / 3$, respectively, and a uniform orientation of 1 . To simplify the discussion here, and considering the bundles' partial orientation in films and meshes' pre-elongation in fibers, we set $\left\langle\cos ^{2} \beta\right\rangle$ to 0.6 for both films and fibers. ${ }^{[21]}$ Table 1 lists the predicted and measured moduli for films and fibers. We can see that the predicted moduli match the measured values very well. Obviously, this method can be applied to other forms of CNT macroarchitectures to predict their moduli. Moreover, since STF is a direct reflection of the microscopic load transfer efficiency and is independent of the CNTs 
Table 1. Measured and predicted moduli from Equation (1) for the films and fibers.

\begin{tabular}{lcccc}
\hline Substrate & $c$ & STF & Predicted modulus [GPa] & Experimental modulus [GPa] \\
\hline CNT films & $0.7-0.8$ & 0.017 & $4.6-5.2$ & $4-6$ \\
CNT fibers & $0.65-0.75$ & 0.045 & $11.2-13$ & $9-15$ \\
\hline
\end{tabular}

volume fraction, we can employ it to evaluate the stiffness of macroscopic CNT assembles without worrying about the indefinite fraction of the void space.

Besides the enhanced downshift rate, the final downshift of the $G^{\prime}$ peak for the CNT fiber $\left(6 \mathrm{~cm}^{-1}\right)$ also increases by about $50 \%$ compared with that for the film $\left(4 \mathrm{~cm}^{-1}\right)$. According to the second piece of analysis presented before, this result shows that the interbundle junctions in the fibers are also reinforced by the twisting process. Since the macroscale fibers' strengths are determined by the strengths of the interbundle junctions, it could be inferred that the fibers have higher strengths than the films. Tensile tests confirm the conclusion from Raman measurements: the strengths of the fibers range from $550-800 \mathrm{MPa}$, in comparison with the strengths of the films of $300-400 \mathrm{MPa}$ (typical strain-stress curves are shown in Fig. 4). Such strengthening of the interbundle junctions could be attributed to the twisting-induced lateral stress in the fibers. Under this type of compressive stress, hollow SWNTs are radially deformed, which generates more friction force to prevent intertube sliding compared with undeformed nanotubes. ${ }^{[22,23]}$ In addition, the long interbundle connections in our directly synthesized films ensure that the friction force could be accumulated to a certain degree to block the free movement of the bundles. ${ }^{[18]}$

The magnitude of the lateral stress in the fiber is related to the geometry of the fiber. We found for a specific piece of film that more twisting resulted in a larger twisting angle, which generated more lateral stress and further strengthened the fabricated fiber.

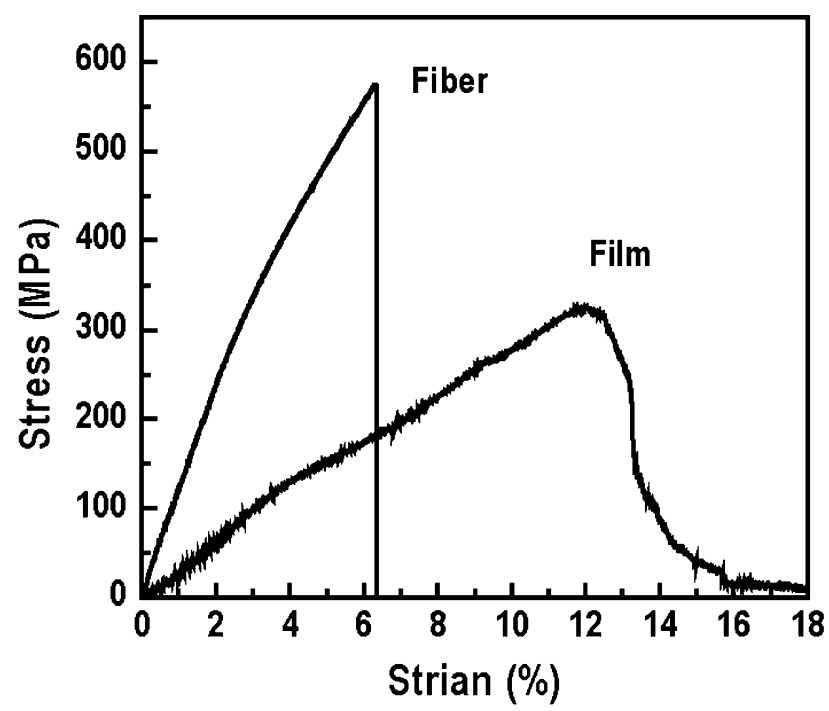

Figure 4. Typical strain-stress curves for the films and fibers. The fracture manner for the fiber turns to catastrophic. It is because the twisting structure blocks the bundles' free sliding and stores more mechanical energy; when a critical defect emerges, the released energy destroys the fiber rapidly.
So to ultimately strengthen the interbundle junctions inside the fibers, we applied twisting as much as possible during the fabrication process, and then found there was twisting a limit for every fiber. Once the twisting limits were reached, the twisting angles stopped increasing with further twsiting, and the fibers started to shrink and weave as a result of excessive torque. The limits of the twisting angle vary with the diameters of the fibers: the thinner fiber usually has a smaller twisting-angle limit. For the fibers with diameters of 30 to $50 \mu \mathrm{m}$, as shown in Figure 5a, there seems a linear relationship between the diameters $r$ and $\sin ^{2} \theta$ ( $\theta$ represents the maximum twisting angle). It could be deduced that the lateral stress near the surface of the fibers was approximately proportional to $\sin ^{2} \theta / r$. So we have good reason to speculate that the lateral stress levels for all of our most-twisted
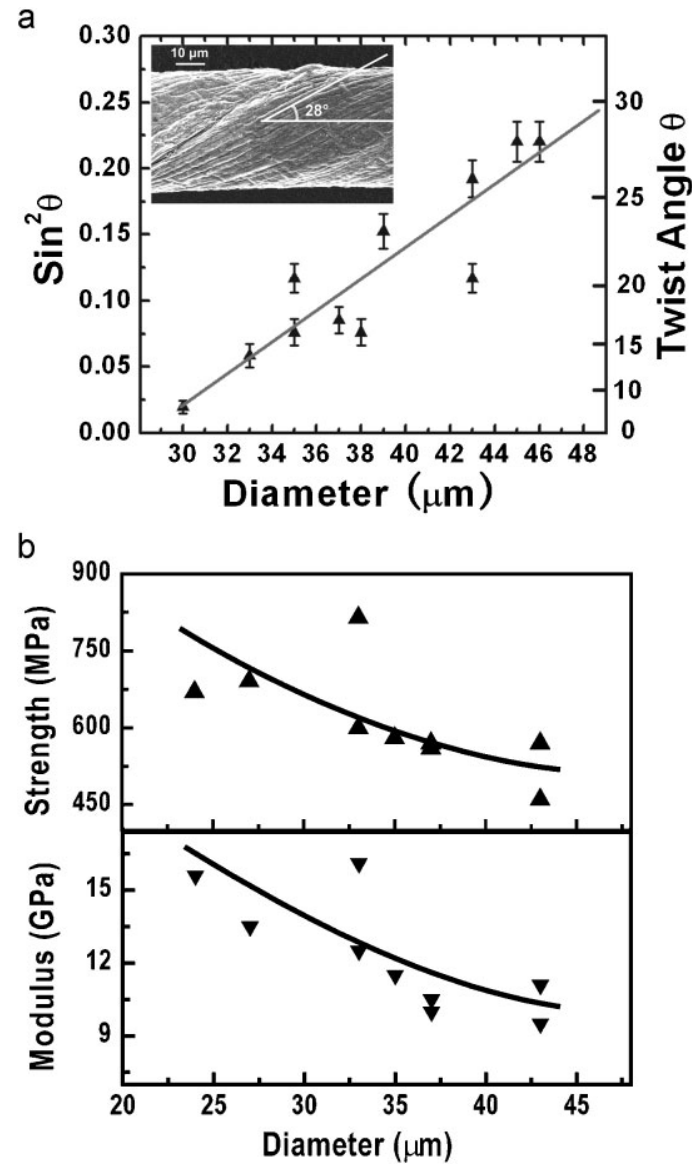

Figure 5. a) Twisting angle-diameter relations for the most-twisted fibers. The inset SEM image shows a fiber with a twisting angle of $28^{\circ}$ and a diameter of $45 \mu \mathrm{m}$. The straight line is plotted to guide the eye. b) Strength-diameter and moduli-diameter relations of the fibers with different diameters. The lines are plotted to guide the eye. 
fibers are similar. To further enhance the lateral stress, stronger films or a new twisting technique may be needed.

Despite that the lateral stress for the CNT fibers with different diameters are similar, the tensile test results show a trend that the strengths and moduli of the fibers decrease as their diameters increase (shown in Fig. 5b). There are other factors in addition to lateral stress that influence the mechanical properties of macroscale CNT fibers. For example, the average incline angles of the SWNTs in thinner fibers are smaller because of smaller twisting angles, which results in a higher moduli; the fibers with a larger diameter have more defects and higher failure possibilities at weak junctions, which aggravates the stress concentration and remarkably weakens the fiber. To further clarify the influences of the fibers' geometries to the micromechanical process, we need more in-situ Raman data that should be recorded during the tensile deformation. But at least, it is clear now, that to obtain strong macroscale CNT fibers, enhancing the lateral stress and reducing the diameter are effective methods.

In summary, by applying Raman tests to strained SWNT films and fibers, the micromechanical process in these CNT macroarchitectures is revealed. We find only a small part of the macroscale strain comes from the axial extension of SWNT bundles, and the strengths of the interbundle junctions determine the strengths of the CNT macroarchitectures. By twisting the SWNT films into fibers, the bundles are pretightened and the junctions are strengthened, so both the moduli and the strengths of the SWNT fibers are promisingly enhanced as a result. Based on the Raman data, we also predict the moduli of the films and fibers, and illustrate the influences of the twisting geometries on the fibers' mechanical performances. We believe this easy and effective methodology can be applied to other kinds of CNT macroarchitectures as a favored supplementary of the macroscale tensile tests to monitor the mechanical process at the micrometer scale.

\section{Experimental}

SWNT films were prepared by a floating catalyst CVD method [18]. Because the as-grown films are not compact, the thicknesses are of meaning only for the densified films. A piece was sliced from each of the films and they were densified by acetone on silicon substrates and the thicknesses subsequently measured by atomic force microscopy (AFM) at the step edges. The thicknesses of the films chosen for twisting were in the range of 150 to $250 \mathrm{~nm}$. We also measured the weight of the films using an ultra-micro balance, and obtained the density of the densified film as $1.3 \pm 0.1 \mathrm{~g} \mathrm{~cm}^{-3}$. By comparing the measured density with that of a closely packed SWNT bundle $\left(1.33 \mathrm{~g} \mathrm{~cm}^{-3}\right)$ and considering the $15-25 \%$ impurity weight fraction in the films, we can conclude that the volume fraction of SWNTs in the film is $0.7-0.8$. (The volume fraction of SWNTs in the fibers was measured by the same method.)

Before the twisting process, no purification process was applied to the SWNT films to protect the natural bonds between the SWNT bundles. During the twisting process, the twisting angles firstly increased with the increased twist, and the fibers became more and more compact. Once the twisting limits were reached, the twisting angles no longer increased, and the fibers started to shrink and weave to balance the excessive torque. The length and diameter of the fabricated fibers were determined by the length and width of an adopted slice of the film. Typically, we synthesized fibers with a length of 4-8 centimeters, and preserved the middle part for tensile tests.
The tensile tests of the films and fibers were performed on an Instron 5848 microtester ( $5 \mathrm{~N}$ load cell). The gauge length was fixed to $1 \mathrm{~cm}$ whether for films or fibers, and the extension rate was $1 \%$ per min The diameters and twisting angles of the fibers were determined from SEM images before performing ensile tests.

\section{Acknowledgements}

This work is supported by National Natural Science Foundation of China (Grant No. 10334060, 50572119, 50753003 and 10572142), '973' National Key Basic Research Program of China (Grant No. 2005CB623602, 2007CB936803), the key item of knowledge innovation project of Chinese Academy of Science KJCX2-YW-M01. One of the authors (L. Song) acknowledges the support of the Alexander von Humboldt Foundation. Supporting Information is available online from Wiley InterScience.

Received: May 14, 2008

Published online: December 12, 2008

[1] M. F. Yu, O. Lourie, M. J. Dyer, K. Moloni, T. F. Kelly, R. S. Ruoff, Science 2000, 287, 637.

[2] M. F. Yu, B. S. Files, S. Arepalli, R. S. Ruoff, Phys. Rev. Lett. 2000, 84, 5552.

[3] G. H. Gao, T. Cagin, W. A. Goddard, Nanotechnology 1998, 9, 184.

[4] R. H. Baughman, A. A. Zakhidov, W. A. de Heer, Science 2002, 297, 787.

[5] L. M. Ericson, H. Fan, H. Q. Peng, V. A. Davis, W. Zhou, J. Sulpizio, Y. H. Wang, R. Booker, J. Vavro, C. Guthy, A. N. G. Parra-Vasquez, M. J. Kim, S. Ramesh, R. K. Saini, C. Kittrell, G. Lavin, H. Schmidt, W. W. Adams, W. Billups, Science 2004, 305, 1447

[6] M. E. Kozlov, R. C. Capps, W. M. Sampson, V. H. Ebron, J. P. Ferraris, R. H. Baughman, Adv. Mater. 2005, 17, 614.

[7] K. L. Jiang, Q. Q. Li, S. S. Fan, Nature 2002, 419, 801.

[8] M. Zhang, K. R. Atkinson, R. H. Baughman, Science 2004, 306, 1358.

[9] X. B. Zhang, K. L. Jiang, C. Teng, P. Liu, L. Zhang, J. Kong, T. H. Zhang, Q. Q. Li, S. S. Fan, Adv. Mater. 2006, 18, 1505.

[10] Y. L. Li, I. A. Kinloch, A. H. Windle, Science 2004, 304, 276.

[11] M. Motta, Y. L. Li, I. Kinloch, A. Windle, Nano Lett. 2005, 5, 1529.

[12] K. Koziol, J. Vilatela, A. Moisala, M. Motta, P. Cunniff, M. Sennett, A. Windle, Science 2007, 318, 1892.

[13] A. M. Rao, E. Richter, S. Bandow, B. Chase, P. C. Eklund, K. A. Williams, S Fang, K. R. Subbaswamy, M. Menon, A. Thess, R. E. Smalley, G. Dresselhaus, M. S. Dresselhaus, Science 1997, 275, 187

[14] H. H. Gommans, J. W. Alldredge, H. Tashiro, J. Park, J. Magnuson, A. G. Rinzler, J. Appl. Phys. 2000, 88, 2509.

[15] S. B. Cronin, A. K. Swan, M. S. Unlu, B. B. Goldberg, M. S. Dresselhaus, M. Tinkham, Phys. Rev. Lett. 2004, 93, 4.

[16] S. B. Cronin, A. K. Swan, M. S. Unlu, B. B. Goldberg, M. S. Dresselhaus, M. Tinkham, Phys. Rev. B 2005, 72, 8.

[17] Q. Zhao, H. D. Wagner, Compos. A 2003, 34, 1219.

[18] W. J. Ma, L. Song, R. Yang, T. H. Zhang, Y. C. Zhao, L. F. Sun, Y. Ren, D. F. Liu, L. F. Liu, J. Shen, Z. X. Zhang, Y. J. Xiang, W. Y. Zhou, S. S. Xie, Nano Lett. 2007, 7, 2307.

[19] X. F. Zhang, Q. W. Li, T. G. Holesinger, P. N. Arendt, J. Y. Huang, P. D. Kirven, T. G. Clapp, R. F. DePaula, X. Z. Liao, Y. H. Zhao, L. X. Zheng, D. E. Peterson, Y. T. Zhu, X. AF Zhang, Q. Li, T. G. Holesinger, P. Arendt, Adv. Mater. 2007, 19, 4198.

[20] T. W. Cheng, W. K. Hsu, Appl. Phy. Lett. 2007, 90, 3.

[21] The CNT bundles' orientation degree in the films or some types of fibers could be determined by polarized Raman measurement (see H.H. 
Gommans, J. W. Alldredge, H. Tashiro, J. Park, J. Magnuson, A. G. Rinzler, Appl. Phys. Lett. 2000, 88, 2509). Since the Raman scattering from the tubes lie near the core of the fibers it is difficult to record, and the exact determination of the orientation degree in the case of our fiber is very difficult to ascertain. To constrain the discussion to the main topic of the communication, here we give a estimated value of 0.6.
[22] D. Qian, W. K. Liu, R. S. Ruoff, Compos. Sci. Technol. 2003, 63, 1561.

[23] X. F. Zhang, Q. W. Li, T. G. Holesinger, P. N. Arendt, J. Y. Huang, P. D. Kirven, T. G. Clapp, R. F. DePaula, X. Z. Liao, Y. H. Zhao, L. X. Zheng, D. E. Peterson, Y. T. Zhu, X. AF Zhang, Q. Li, T. G. Holesinger, P. Arendt, Adv. Mater. 2007, 19, 4198. 